1 Hacettepe Journal of Mathematics and Statistics

$\bigcap$ Volume 47 (4) (2018), 845-854

\title{
Coverings of structured Lie groupoids
}

\author{
M. Habil Gürsoy*† and İlhan İcen ${ }^{\ddagger}$
}

\begin{abstract}
In this work, we present some results related to coverings of structured Lie groupoids. Firstly, we obtain a covering Lie group-groupoid and a covering morphism of Lie group-groupoids from a given Lie groupgroupoid by the notion of action. Secondly, we show how the Lie group structure of a Lie group-groupoid is lifted to a covering Lie groupoid. Then, we give similar results for Lie ring-groupoid which is also a structured Lie groupoid.
\end{abstract}

Keywords: Lie Groupoid, Covering, Structured Lie Groupoid.

Mathematics Subject Classification (2010): 22A22; 57M10

Received : 23.08.2016 Accepted : 29.05.2017 Doi : 10.15672/HJMS.2017.483

\section{Introduction}

Covering spaces are used extensively in many branches of mathematics. The reflection of the concept of covering space is the concept of covering groupoid in the groupoid theory. First papers in this area were studied by Brown and Higgins. After presenting fundamental groupoid $\pi_{1} X$ for a topological space $X$, Brown has defined the concept of covering morphism between the fundamental groupoids corresponding to a covering map between the topological spaces. Then he has also shown that the category of coverings of topological space $X$ having a universal covering is equivalent to that of coverings of fundamental groupoid $\pi_{1} X[1]$.

There is another important paper on covering groupoids which was introduced to the literature by Gabriel and Zisman. Gabriel and Zisman proved that the category of the actions on sets of a groupoid $G$ is equivalent to that of the covering groupoids of $G$ [5].

\footnotetext{
*İnönü University, Faculty of Science and Art, Department of Mathematics, 44280, MalatyaTURKEY,

${ }^{\ddagger}$ Inonu University, Faculty of Science and Art, Department of Mathematics, 44280, MalatyaTURKEY,

Email : ilhan.icen@inonu.edu.tr
} 
In 1956, Ehresmann introduced the concepts of topological and Lie groupoid. As a consequence of this, several algebraic papers are written concerning topological and Lie groupoids $[2,8]$.

Structured groupoids, subject of this work, are special groupoids which have another algebraic structure such as group structure or ring structure as well as groupoid structure. Firstly, the concept of group-groupoid was introduced by Brown and Spencer in [3]. Brown and Spencer have proved that the fundamental groupoid corresponding to a topological group $X$ is a group-groupoid.

Another structured groupoid covered in the present study is ring-groupoid. The concept of ring-groupoid was presented by Mucuk [12]. Mucuk has proved how the ring structure of a topological ring $X$ is lifted. Additionally, he has also shown the category of the coverings of topological ring $X$ is equivalent to that of the coverings of fundamental

Lie ring groupoid $\pi_{1} X$ corresponding to $X$.

The topological versions of these papers are given in $[13,9]$.

A structured Lie groupoid is a Lie groupoid having another Lie structure such as Lie group or Lie ring. In this work, by a structured Lie groupoid we are going to mean Lie groupoids having Lie group and Lie ring structures, and are going to give some results about the coverings of structured Lie groupoids. We show how a covering structured Lie groupoid and a covering morphism of structured Lie groupoids from a given structured Lie groupoid are obtained. Moreover, we show that a covering groupoid of a transitive Lie group-groupoid, resp. Lie ring-groupoid, inherits the structure of a Lie group-groupoid, resp. Lie ring-groupoid.

All manifolds under consideration in this work are connected, smooth, Hausdorff and second countable.

\section{Structured Lie Groupoids}

Structured groupoids are special groupoids which have another algebraic structure such as group structure or ring structure as well as groupoid structure. Similarly, by a structured Lie groupoid, we mean a Lie groupoid having another Lie structure. In this work, we deal with Lie group-groupoids and Lie ring-groupoids which are structured Lie groupoids. This section of the paper is devoted to give basic definitions and concepts of the structured Lie groupoids. Firstly, we recall some definitions to fix our notation.

2.1. Definition. A Lie groupoid $G$ over object set $G_{0}$ is a groupoid whose set of objects and arrows are both manifolds, the source map $\alpha$, the target map $\beta$, the object map $\epsilon$, the partial multiplication $m$ and the inversion $i$ are all smooth maps and such that $\alpha$ and $\beta$ are surjective submersions which implies that the set of composable arrows is a submanifold of $G \times G[10,11]$.

Let $G \rightrightarrows G_{0}$ be a Lie groupoid. By $G(x, y)$, we denote the set of arrows such that $\alpha(a)=x$ and $\beta(a)=y$ for all $x, y \in G_{0}$. The set $S t_{G} x$ of arrows starting at $x$ and the set $\operatorname{CoSt}_{G} x$ of arrows finishing at $x$ are called the $\alpha$-fibre and $\beta$-fibre at $x \in G_{0}$, respectively. The sets $G(x, y), S t_{G} x$ and $\operatorname{CoSt}_{G} x$ are embedded closed submanifolds of $G$. The isotropy group at $x$ is $G(x)=\{a \in G \mid \alpha(a)=\beta(a)=x\}$, which is a Lie group. If there is at least one arrow in $G(x, y)$, a Lie groupoid $G$ is transitive[1].

The left-translation (right-translation) corresponding to $a \in G(x, y)$ is a diffeomorphism $L_{a}: \operatorname{CoSt}_{G} x \rightarrow \operatorname{CoSt}_{G} y, b \mapsto a \circ b\left(R_{a}: S t_{G} y \rightarrow S t_{G} x, b \mapsto b \circ a\right)$. [10].

2.2. Definition. A homomorphism of Lie groupoids $H$ and $G$ is a groupoid homomorphism $\left(f, f_{0}\right)$ such that $f$ and $f_{0}$ are smooth $[10,11]$.

Now, let us recall some examples which we need in the paper: 
2.3. Examples. (1) Banal Lie Groupoid: The product $M \times M$ is a Lie groupoid with object manifold $M$.

(2) Action Lie Groupoid: Any Lie group $G$ acting on a manifold $M$ determines a Lie groupoid with the composition $(b, a \cdot x)(a, x)=(b a, x)$ for all $a, b \in G$ and $x \in M$.

Now, let us give definition of a Lie group-groupoid.

2.4. Definition. A Lie group-groupoid is a Lie groupoid having a structure of Lie group such that the structure maps of the Lie group are Lie groupoid morphisms. Also there exists an interchange law which gives the compatibility between the group operation and the groupoid composition, i.e.,

$$
(b \circ a)+(d \circ c)=(b+d) \circ(a+c)[6] .
$$

2.5. Definition. A homomorphism $f: H \rightarrow G$ of Lie group-groupoids is a Lie groupoid homomorphism preserving the Lie group structure [6].

2.6. Example. Given a Lie group $G$, we constitute a Lie group-groupoid $G \times G$ with object manifold $G$ as follows.

The structure of groupoid:

a morphism is a pair $(y, x)$ in $G \times G$.

the source map: $\alpha(y, x)=x$,

the target map: $\beta(y, x)=y$,

the object map: $\epsilon(x)=(x, x)$ for any $x \in G$,

the inverse map: $i(y, x)=(x, y)$,

the composition: $(z, y) \circ(y, x)=(z, x)$

The structure of group:

the group operation: $(x, y)+(z, t)=(x+z, y+t)$

the unit element of $G \times G:(e, e)$, where $e$ is the unit element of $G$, the inverse in the group of $(y, x):(-y,-x)$

The interchange law:

$$
\begin{aligned}
((z, y) \circ(y, x))+\left(\left(z^{\prime}, y^{\prime}\right) \circ\left(y^{\prime}, x^{\prime}\right)\right) & =(z, x)+\left(z^{\prime}, x^{\prime}\right) \\
& =\left(z+z^{\prime}, x+x^{\prime}\right)
\end{aligned}
$$

and

$$
\begin{aligned}
\left((z, y)+\left(z^{\prime}, y^{\prime}\right)\right) \circ\left((y, x)+\left(y^{\prime}, x^{\prime}\right)\right) & =\left(z+z^{\prime}, y+y^{\prime}\right) \circ\left(y+y^{\prime}, x+x^{\prime}\right) \\
& =\left(z+z^{\prime}, x+x^{\prime}\right)
\end{aligned}
$$

From (2.1) and (2.2), it is seen that the interchange law holds.

Furthermore, it is easy to show that the group structure maps of $G \times G$ are groupoid morphisms.

Since the group structure maps of $G \times G$ are defined by the operations of Lie group $G$, they are also smooth. Consequently, $G \times G$ is a Lie group-groupoid [6].

The transitivity of a Lie group-groupoid $G$ is defined via transitivity of the underlying Lie groupoid of $G$.

Now let us recall definition of Lie ring which is a basic notion in the structure of Lie ring-groupoid.

2.7. Definition. A Lie ring $R$ is a ring $R$ whose underlying set has a structure of manifold such that the group operation $m: R \times R \rightarrow R,(x, y) \mapsto x+y$, the ring operation $n: R \times R \rightarrow R,(x, y) \mapsto x y$ and the inverse map $u: R \rightarrow R, x \mapsto-x$ are smooth [6]. 
A Lie ring homomorphism is a ring homomorphism which is smooth at the same time. Hence, there exists a category $L$ Ring $(R)$ of Lie rings $R$. Objects of this category are Lie rings $R$, and its morphisms are Lie ring morphisms.

Let $p: H \rightarrow R$ be a Lie ring homomorphism. If $p$ is a smooth covering map of underlying manifolds of $H$ and $R$, then $p$ is called a covering morphism of Lie rings.

Further, let $\widetilde{R}$ and $R$ be connected Lie rings. Here connected Lie ring means that a ring whose manifold structure is connected. Then, we have a category $L R C o v(R)$ of smooth coverings of connected Lie ring $R$.

The definition of a Lie ring-groupoid is as follows:

2.8. Definition. A Lie ring-groupoid $R$ is a Lie groupoid which is a Lie ring at the same time such that following ring structure maps are Lie groupoid homomorphisms.

i) $m: R \times R \rightarrow R,(a, b) \mapsto a+b$, group operation

ii) $n: R \times R \rightarrow R,(a, b) \mapsto a b$, ring operation

iii) $u: R \rightarrow R, a \mapsto-a$, inverse in group

iv) $e: * \rightarrow R$.

Also there exist following interchange laws in a Lie ring-groupoid $R$.

(1) $(c \circ a)+(d \circ b)=(c+d) \circ(a+b)$,

(2) $(c \circ a)(d \circ b)=(c d) \circ(a b)[6]$.

A Lie ring-groupoid homomorphism is a Lie groupoid homomorphism preserving the Lie ring structure.

2.9. Example. Given a Lie ring $R$, we can construct a Lie ring-groupoid $R \times R$ over the object manifold $R$. In this Lie ring-groupoid we define the ring operation by $(a, b)(c, d)=$ $(a c, b d)$ for all $a, b, c, d \in R$ (for more details, see [6]).

The transitivity of a Lie ring-groupoid $R$ is defined via transitivity of the underlying Lie groupoid of $R$.

\section{Coverings of Structured Lie Groupoids}

It will be useful to present the definition of covering morphism of Lie groupoids before the definitions of covering morphisms of structured Lie groupoids.

3.1. Definition. Let $p: \widetilde{G} \rightarrow G$ be a Lie groupoid homomorphism. For each $\widetilde{x} \in \widetilde{G}_{0}$, if the restriction $\widetilde{G}_{\widetilde{x}} \rightarrow G_{p(\widetilde{x})}$ of $p$ is a diffeomorphism, $p$ is called the covering morphism of Lie groupoids. Then Lie groupoid $\widetilde{G}$ is also called the covering of the Lie groupoid $G$ [8].

Let us give a criterion for a morphism of Lie groupoids to be a covering morphism of Lie groupoids.

Let $p: H \rightarrow G$ be a covering morphism of Lie groupoids. Take the pullback

$$
G_{\alpha} \times_{p_{0}} H_{0}=\left\{(a, x) \in G \times H_{0} \mid \alpha(a)=p_{0}(x)\right\} .
$$

Since $\alpha$ is a submersion, $G{ }_{\alpha} \times{ }_{p_{0}} H_{0}$ is a manifold. Then the map $s_{p}: G{ }_{\alpha} \times{ }_{p_{0}} H_{0} \rightarrow H$ is the lifting function assigned to the unique element $h \in H_{x}$, the pair $(a, x)$ such that $p(h)=a$. The map $s_{p}$ is the inverse of the map $(p, \alpha): H \rightarrow G{ }_{\alpha} \times_{p_{0}} H_{0}$.

Thus the homomorphism $p: H \rightarrow G$ is a covering morphism of Lie groupoids if and only if the morphism $(p, \alpha)$ is a diffeomorphism.

3.2. Definition. A morphism $f: H \rightarrow G$ of Lie group-groupoids is called a covering morphism of Lie group-groupoids if it is a covering morphism of underlying Lie groupoids [6].

Let us now recall a proposition without proof from [1] which is necessary for the proof of the Theorem 3.4. 
3.3. Proposition. Let $p: \widetilde{G} \rightarrow G$ be a covering morphism of groupoids, $x \in G_{0}$ and $\widetilde{x} \in \widetilde{G}_{0}$ such that $p(\widetilde{x})=x$. Let $q: H \rightarrow G$ be a groupoid homomorphism such that $H$ is transitive and $\widetilde{y} \in H_{0}$ such that $q(\widetilde{y})=x$. Then the homomorphism $q: H \rightarrow G$ uniquely lifts to a homomorphism $\widetilde{q}: H \rightarrow \widetilde{G}$ such that $\widetilde{q}(\widetilde{y})=\widetilde{x}$ if and only if $q[H(\widetilde{y})] \subseteq p[\widetilde{G}(\widetilde{x})]$, where $H(\widetilde{y})$ and $\widetilde{G}(\widetilde{x})$ are the isotropy groups.

Now, let us give a theorem which denotes how Lie group structure of a Lie groupgroupoid is lifted to a covering Lie groupoid.

3.4. Theorem. Let $\widetilde{G}$ be a transitive Lie groupoid and let $G$ be a transitive Lie groupgroupoid. Let $p: \widetilde{G} \rightarrow G$ be covering morphism on the underlying Lie groupoids. Let e be the identity element of $G_{0}$ and $\widetilde{e} \in \widetilde{G}_{0}$ such that $p(\widetilde{e})=e$. Then the Lie group structure of $G$ lifts to $\widetilde{G}$ with identity $\widetilde{e}$ in such a way that $\widetilde{G}$ becomes a Lie group-groupoid.

Proof. Since $G$ is a Lie group-groupoid, there exist the following maps:

$$
\begin{gathered}
m: G \times G \rightarrow G,(a, b) \mapsto a+b, \\
u: G \rightarrow G, a \mapsto-a .
\end{gathered}
$$

Since $\widetilde{G}$ is the covering groupoid of $G$, the isotropy group $\widetilde{G}(\widetilde{e})$ has one element at most. Thus, by Proposition 3.3, the above maps lift to the maps

$$
\begin{gathered}
\widetilde{m}: \widetilde{G} \times \widetilde{G} \rightarrow \widetilde{G},(\widetilde{a}, \widetilde{b}) \mapsto \widetilde{a}+\widetilde{b} \\
\widetilde{u}: \widetilde{G} \rightarrow \widetilde{G}, \widetilde{a} \mapsto-\widetilde{a}
\end{gathered}
$$

by $p: \widetilde{G} \rightarrow G$, respectively such that

$$
\begin{gathered}
p(\widetilde{a}+\widetilde{b})=p(\widetilde{a})+p(\widetilde{b}), \\
p(-\widetilde{a})=-p(\widetilde{a}) .
\end{gathered}
$$

Since the addition $m: G \times G \rightarrow G,(a, b) \mapsto a+b$ is associative, we have $m \times(m \times 1)=$ $m \times(1 \times m)$, where 1 denotes the identity map. By Proposition 3.3, the maps $m \times(m \times 1)$ and $m \times(1 \times m)$, respectively, lift to

$$
\widetilde{m} \times(\widetilde{m} \times 1), \widetilde{m} \times(1 \times \widetilde{m}): \widetilde{G} \times \widetilde{G} \times \widetilde{G} \rightarrow \widetilde{G}
$$

which coincide at $(\widetilde{e}, \widetilde{e}, \widetilde{e})$. By the uniqueness of the lifting, we have $\widetilde{m} \times(\widetilde{m} \times 1)=$ $\widetilde{m} \times(1 \times \widetilde{m})$, i.e., $\widetilde{m}$ is associative. In the similar way, we can show that $\widetilde{e}$ is the identity and $-\widetilde{a}$ is the inverse arrow of $\widetilde{a}$.

Now, let us prove that the group operation is smooth.

Since $G$ is connected manifold, it has a universal covering manifold. Hence we can choose an atlas $\mathcal{A}$ of liftable coordinate charts of $G$. Then there exists a lifted atlas on $\widetilde{G}$, because $p: \widetilde{G} \rightarrow G$ is a covering morphism of underlying Lie groupoids. Let us denote this lifted atlas by $\widetilde{\mathcal{A}}$. Let $\widetilde{U}$ be a coordinate chart of $\widetilde{e}$ and a lifting of $U \in \mathcal{A}$. Since $m$ is smooth, there exists a coordinate chart of $e$ in $G$ such that $m(V \times V) \subseteq U$. If we use the condition on $G$ and choose $V$ small enough, then we can suppose that $V$ is a simply connected coordinate chart. Let $\widetilde{V}$ be lifting of $V$ in the Lie groupoid $\widetilde{G}$. Then $p(\widetilde{m}(\widetilde{V} \times \widetilde{V}))=m(V \times V) \subseteq U$ and so we have $\widetilde{m}(\widetilde{V} \times \widetilde{V}) \subseteq \widetilde{U}$. Thus, the smoothness of the map

$$
\widetilde{m}: \widetilde{G} \times \widetilde{G} \rightarrow \widetilde{G},(\widetilde{a}, \widetilde{b}) \mapsto \widetilde{a}+\widetilde{b}
$$

follows. Therefore, $\widetilde{G}$ becomes a Lie group-groupoid and $p$ is clearly a Lie group-groupoid homomorphism. 
In Theorem 3.10, we will investigate the relation between covering of a Lie groupgroupoid and action of a Lie group-groupoid on a Lie group. But for this, we have to recall the action of a Lie groupoid on a manifold and then have to give the definition of the action of a Lie group-groupoid on a Lie group.

3.5. Definition. A left action of a Lie groupoid $G$ on a manifold $M$ via a submersion $w: M \rightarrow G_{0}$ is a smooth map $\phi: G_{\alpha \times}{ }_{w} M \rightarrow M,(a, x) \mapsto a: x$ such that the following conditions hold:

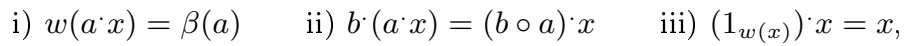
for any $a, b \in G, x \in M$. The right action is defined similarly [11].

In general, we will use the notation $a: x$ for the action, but sometimes we might use the notation ${ }^{a} x$ as well.

3.6. Example. Let $p: H \rightarrow G$ be a covering morphism of Lie groupoids. We take $M=$ $H_{0}$ and $w=p_{0}: H_{0} \longrightarrow G_{0}$. Thus, we obtain the smooth action $\phi: G_{\alpha \times p_{0}} H_{0} \rightarrow H_{0}$, $(a, \widetilde{x}) \mapsto a \cdot \widetilde{x}=\widetilde{\beta}(\widetilde{a})$ of $G$ on $M=H_{0}$ by $w=p_{0}$ (for more details, see [8]).

3.7. Example. Let $G$ be a Lie groupoid acting on manifold $M$ via a submersion $w$ : $M \rightarrow G_{0}$. By this action, we can define a Lie groupoid denoted by $G \ltimes M$ which is called the action Lie groupoid [8].

3.8. Definition. An action of Lie group-groupoid $G$ on Lie group $M$ via Lie group homomorphism $w: M \rightarrow G_{0}$ consists of the action of the underlying Lie groupoid of $G$ on the underlying manifold of $M$ via the submersion $w: M \rightarrow G_{0}$ such that interchange law $\left({ }^{b} y\right)+\left({ }^{a} x\right)={ }^{b+a}(y+x)$ holds. Such an action is denoted by $(M, w)[6]$.

3.9. Example. A Lie group-groupoid $G$ acts on manifold $M=G_{0}$ via the unit morphism $w=p_{0}: M=G_{0} \rightarrow G_{0}$ (for more details, see [6]).

Now, let us prove a theorem showing how a covering Lie group-groupoid and a smooth covering morphism of Lie group-groupoids from a given Lie group-groupoid are obtained. In the following theorem, $N(e)$ denotes the closed subgroup of the object group $G(e)$ of the unit element $e$. We define the set $M=\left\{a \circ N(e) \mid a \in S t_{G} e\right\}$. In fact, the set $M$ has a group structure with the operation $(a \circ N(e))+(b \circ N(e))=(a+b) \circ N(e)$, where $a, b \in S t_{G} e$. Since the operation on $M$ is defined by the operation of Lie group $G$ and the composition of the Lie groupoid, it is smooth. The unit element of $M$ is $1_{e} \circ N(e)$ and inverse of $a \circ N(e)$ is $-a \circ N(e)$, where $-a$ is the inverse of $a$ in Lie group $G$.

3.10. Theorem. Let $G$ be a transitive Lie group-groupoid with the unit element e. Then the action groupoid $H=G \ltimes M$ is naturally a Lie group-groupoid with the identity element $N(e)$. Furthermore, there exists a natural covering morphism $p: H \rightarrow G$ such that $p(H(\widetilde{e}))=N(e)$.

Proof. Let us define $w: M \rightarrow G_{0}$ by $a \circ N(e) \mapsto \beta(a)$. Then

$$
\begin{aligned}
w((a \circ N(e))+(b \circ N(e))) & =w(a+b \circ N(e)) \\
& =\beta(a+b)=\beta(a)+\beta(b) \\
& =w(a \circ N(e))+w(b \circ N(e)) .
\end{aligned}
$$

$w: M \rightarrow G_{0}$ is a group homomorphism, and $w$ is clearly a smooth submersion. We define action by

$$
\phi: G_{\alpha} \times_{w} M \rightarrow M,(b, a \circ N(e)) \mapsto b \circ a \circ N(e) .
$$

Now let us check the action conditions: 
Firstly, we have $\alpha(b)=w(a \circ N(e))=\beta(a)$ for $a \in G(e, y)$ and $b \in G(y, z)$. So $b \circ a$ is defined and $b \circ a \in G_{e}$. Hence, we have

$$
w\left({ }^{b}(a \circ N(e))\right)=w(b \circ a \circ N(e))=\beta(b \circ a)=\beta(b) .
$$

That is, the first condition is satisfied.

Secondly, if $c \in G\left(z, z^{\prime}\right)$, then

$$
\begin{aligned}
{ }^{c}\left({ }^{b}(a \circ N(e))\right) & ={ }^{c}(b \circ a \circ N(e)) \\
& =c \circ(b \circ a \circ N(e)) \\
& =(c \circ b) \circ(a \circ N(e)) \\
& ={ }^{(c \circ b)}(a \circ N(e)) .
\end{aligned}
$$

That is, the second condition is satisfied.

Finally, since

$$
{ }^{1} y(a \circ N(e))=1_{y} \circ a \circ N(e)=a \circ N(e),
$$

the third condition is also satisfied.

Smoothness of the action $\phi$ is similar to that in the case of Lie groupoids given in [8]. Thus, we obtain a Lie group-groupoid $G \ltimes M$ whose objects manifold is $(G \ltimes M)_{0}=M$. A morphism from an object $a \circ N(e)$ to another one $a^{\prime} \circ N(e)$ in this group-groupoid is a pair $(b, a \circ N(e))$ such that $^{b}(a \circ N(e))=a^{\prime} \circ N(e)$, where $b \circ a=a^{\prime}$. The composition is defined by $(b, a \circ N(e)) \circ(c, a \circ N(e))=(b \circ c, a \circ N(e))$. Also, $G \ltimes M$ is a Lie group with group operation

$$
\left(b, a^{\prime} \circ N(e)\right)+(c, a \circ N(e))=\left(b+c,\left(a^{\prime}+a\right) \circ N(e)\right)
$$

and the structure of manifold induced from the product manifold $G \times M$. Unit element of this group is $\left(1_{e}, 1_{e} \circ N(e)\right)$ and inverse of $(b, a \circ N(e))$ is $(-b,-a \circ N(e))$, where $-b$ and $-a$ are inverses of $b$ and $a$ in the group. It is obvious smoothness of the groupoid structure maps by [8]. Thus, $G \ltimes M$ is a Lie group-groupoid.

Let $p: G \ltimes M \rightarrow G$ be defined by $(b, a \circ N(e)) \mapsto b$ on morphisms and by $w$ on objects. $p_{0}$, is given by $w$, is a homomorphism of Lie groups. Since $p$ is the projection, it is smooth. The map $p$ is a groupoid homomorphism, because

$$
\begin{aligned}
p\left(\left(b, a^{\prime} \circ N(e)\right) \circ(c, a \circ N(e))\right) & =p(b \circ c, a \circ N(e)) \\
& =b \circ c \\
& =p\left(b, a^{\prime} \circ N(e)\right) \circ p(c, a \circ N(e)) .
\end{aligned}
$$

In addition, it preserves the group structure, namely

$$
\begin{aligned}
p\left(\left(b, a^{\prime} \circ N(e)\right)+(c, a \circ N(e))\right) & =p\left(b+c,\left(a^{\prime}+a\right) \circ N(e)\right) \\
& =b+c \\
& =p\left(b, a^{\prime} \circ N(e)\right)+p(c, a \circ N(e)) .
\end{aligned}
$$

Thus, $p$ is a Lie group-groupoid homomorphism.

Since $p_{0}=w$ and $(G \ltimes M)_{0}=M$, lifting map $s_{p}: G \alpha_{\alpha} \times_{p_{0}} M \rightarrow G \ltimes M$ is unit map. So it is one-to-one, surjective and smooth. Inverse map $(p, \alpha): G \ltimes M \rightarrow G{ }_{\alpha} \times_{p_{0}} M$ of $s_{p}$ is smooth, because $p$ is smooth and $\alpha$ is the source map of Lie group-groupoid. Thus $s_{p}$ is a diffeomorphism. Consequently, $p: G \ltimes M \rightarrow G$ is a covering morphism of Lie group-groupoids. If we take $H=G \ltimes M$ and $\widetilde{x}=N(x)$, we have $p(H(\widetilde{x}))=N(x)$.

Let us now remember notion of covering of Lie ring-groupoids. 
3.11. Definition. Let $p: \widetilde{R} \rightarrow R$ be a Lie ring-groupoid homomorphism. $p$ is called a covering morphism of Lie ring-groupoids, if it is a covering morphism of underlying Lie groupoids of $\widetilde{R}$ and $R$. In other words, if the morphism $s_{p}: R{ }_{\alpha} \times_{p_{0}} \widetilde{R}_{0} \rightarrow \widetilde{R}$ defined on underlying Lie groupoids of $\widetilde{R}$ and $R$ is a diffeomorphism, then $p$ is called covering morphism of Lie ring-groupoids. The inverse of $s_{p}$ is $(p, \alpha): \widetilde{R} \rightarrow R{ }_{\alpha} \times{ }_{p_{0}} \widetilde{R}_{0}$ [6].

3.12. Example. Let $p: R \rightarrow R$ be unit homomorphism of Lie ring-groupoids. Then, it is clear that projection $s_{p}: R{ }_{\alpha} \times_{p_{0}} R_{0} \rightarrow R$ is one-to-one, surjective and smooth. Further, $p$ and $\alpha$ are smooth, because $p$ is the Lie ring-groupoid homomorphism and $\alpha$ is the source map of Lie ring-groupoid $R$. Hence the inverse $(p, \alpha): R \rightarrow R{ }_{\alpha} \times_{p_{0}} R_{0}$ of $s_{p}$ is smooth. Therefore, $s_{p}$ is a diffeomorphism. Consequently, $p$ is a covering morphism of Lie ring-groupoids [6].

Now, let us show how the Lie ring structure of a Lie ring-groupoid is lifted.

3.13. Theorem. Let $\widetilde{R}$ be a transitive Lie groupoid and $R$ be a transitive Lie ringgroupoid. Let $p: \widetilde{R} \rightarrow R$ be a universal covering morphism of the underlying Lie groupoids. Let 0 be zero element of Lie ring $R_{0}$ and $\widetilde{0} \in \widetilde{R}_{0}$ such that $p(\widetilde{0})=0$. Under all these conditions, the Lie ring structure of $R$ lifts to $\widetilde{R}$ with the zero element $\widetilde{0}$ in such a way that $\widetilde{R}$ becomes a Lie ring-groupoid.

Proof. Since $R$ is a Lie ring-groupoid, we have the following maps:

$$
\begin{array}{rll}
m: R \times R \rightarrow R & , & (a, b) \mapsto a+b \\
n: R \times R \rightarrow R & , \quad(a, b) \mapsto a b \\
u: R \rightarrow R & , \quad a \mapsto-a \\
0: * \rightarrow R . &
\end{array}
$$

The isotropy group $\widetilde{R}(\widetilde{0})$ has at most one element, because $\widetilde{R}$ is a universal covering Lie groupoid. Thus, from Proposition 3.3, the above maps lift to the maps

$$
\begin{array}{rll}
\widetilde{m}: \widetilde{R} \times \widetilde{R} \rightarrow \widetilde{R} & , \quad & (\widetilde{a}, \widetilde{b}) \mapsto \widetilde{a}+\widetilde{b} \\
\widetilde{n}: \widetilde{R} \times \widetilde{R} \rightarrow \widetilde{R} & , \quad(\widetilde{a}, \widetilde{b}) \mapsto \widetilde{a} \widetilde{b} \\
& \widetilde{u}: \widetilde{R} \rightarrow \widetilde{R} \quad, \quad \widetilde{a} \mapsto-\widetilde{a} \\
\widetilde{0}: \widetilde{*} \rightarrow \widetilde{R}, &
\end{array}
$$

respectively by $p: \widetilde{R} \rightarrow R$ such that for any $\widetilde{a}, \widetilde{b} \in \widetilde{R}$,

$$
\begin{aligned}
p(\widetilde{a}+\widetilde{b}) & =p(\widetilde{a})+p(\widetilde{b}), \\
p(\widetilde{a} \tilde{b}) & =p(\widetilde{a}) p(\widetilde{b}), \\
p(\widetilde{u}(\widetilde{a})) & =-p(\widetilde{a}) .
\end{aligned}
$$

Since the multiplication $m: R \times R \rightarrow R,(a, b) \mapsto a+b$ is associative, we have $m \times(m \times 1)=m \times(1 \times m)$, where 1 denotes the identity map. Then, again by Proposition 3.3 , the maps $m \times(m \times 1)$ and $m \times(1 \times m)$, respectively, lift to

$$
\widetilde{m} \times(\widetilde{m} \times 1), \widetilde{m} \times(1 \times \widetilde{m}): \widetilde{R} \times \widetilde{R} \times \widetilde{R} \rightarrow \widetilde{R}
$$

which coincide at $(\widetilde{0}, \widetilde{0}, \widetilde{0})$. By the uniqueness of the lifting, we have $\widetilde{m} \times(\widetilde{m} \times 1)=$ $\widetilde{m} \times(1 \times \widetilde{m})$, i.e., $\widetilde{m}$ is associative. Similarly, $\widetilde{n}$ is associative. In a similar way, we can show that $\widetilde{0}$ is the zero element and $-\widetilde{a}$ is the inverse element of $\widetilde{a}$. Now, let us show that the group multiplication

$$
\widetilde{m}: \widetilde{R} \times \widetilde{R} \rightarrow \widetilde{R},(\widetilde{a}, \widetilde{b}) \mapsto \widetilde{a}+\widetilde{b}
$$


is smooth.

By assuming that $R$ has a universal covering, we can choose an atlas $\mathcal{A}$ of liftable coordinate charts of $R$. Since $p$ is a local diffeomorphism, the atlas on $\widetilde{R}$ obviously consists of all liftings of the coordinate charts in $\mathcal{A}$. Let $\widetilde{U}$ be an open neighborhood of $\widetilde{0}$ and lifting of $U \in \mathcal{A}$. Since the multiplication $m: R \times R \rightarrow R,(a, b) \mapsto a+b$ is smooth, there is an open neighborhood $V$ of 0 in $R$ such that $m(V \times V) \subseteq U$. Using the condition on $R$ and choosing $V$ small enough, we can assume that $V$ is liftable. Let $\widetilde{V}$ be the lifting of $V$. Then $p(\widetilde{m}(\widetilde{V} \times \widetilde{V}))=m(V \times V) \subseteq U$ and so we have $\widetilde{m}(\widetilde{V} \times \widetilde{V}) \subseteq \widetilde{U}$. Hence $\widetilde{m}$ is smooth. Similarly, $\widetilde{n}$ is smooth. Further, the distributive law is satisfied as follows: Let $p_{1}, p_{2}: R \times R \times R \rightarrow R$ be morphisms defined by

$$
p_{1}(a, b, c)=a b \quad, \quad p_{2}(a, b, c)=b c
$$

and

$$
\left(p_{1}, p_{2}\right): R \times R \times R \rightarrow R \times R,(a, b, c) \mapsto(a b, b c)
$$

for $a, b, c \in R$. Since the distributive law is satisfied in $R$, we have $n \times(1 \times m)=$ $m \times\left(p_{1}, p_{2}\right)$. The maps $n \times(1 \times m)$ and $m \times\left(p_{1}, p_{2}\right)$, respectively, lift to the maps

$$
\widetilde{n} \times(1 \times \widetilde{m}), \widetilde{m} \times\left(\widetilde{p}_{1}, \widetilde{p}_{2}\right): \widetilde{R} \times \widetilde{R} \times \widetilde{R} \rightarrow \widetilde{R}
$$

coinciding at $(\widetilde{0}, \widetilde{0}, \widetilde{0})$. So by Proposition 3.3 , we have $\widetilde{n} \times(1 \times \widetilde{m})=\widetilde{m} \times\left(\widetilde{p}_{1}, \widetilde{p}_{2}\right)$. It means that the distribution law on $\widetilde{R}$ is satisfied. Hence, $\widetilde{R}$ becomes a Lie ring-groupoid and $p$ is clearly a Lie ring-groupoid homomorphism.

In the Theorem 3.16, we present a method to obtain a covering Lie ring-groupoid by the concept of action. For this reason, we have to recall the following definition from [6].

3.14. Definition. An action of the Lie ring-groupoid $R$ on the Lie $\operatorname{ring} M$ via smooth ring homomorphism $w: M \rightarrow R_{0}$ is a smooth map $\phi: R_{\alpha} \times_{w} M \rightarrow M$ satisfying the conditions $w\left({ }^{a} x\right)=\beta(a),{ }^{b}\left({ }^{a} x\right)={ }^{b \circ a} x$ and ${ }^{1} w(x) x=x$ such that the Lie groupoid $R$ acts smoothly on the manifold $M$ via smooth submersion $w: M \rightarrow R_{0}$ and the followings are hold:

(1) $\left({ }^{b} y\right)+\left({ }^{a} x\right)={ }^{b+a}(y+x)$,

(2) $\left({ }^{b} y\right)\left({ }^{a} x\right)={ }^{b a}(y x)[6]$.

3.15. Example. If $R$ is a Lie ring-groupoid, then $R$ acts smoothly on the Lie ring $M=R_{0}$ via the unit morphism $w=p_{0}: R_{0} \rightarrow R_{0}$. The action is defined by $\phi:$ $R{ }_{\alpha} \times{ }_{w} R_{0} \rightarrow R_{0}, \phi(a, x)={ }^{a} x=\beta(a)$ for any $a \in R$ and $x \in R_{0}$ (for more details, see [6]).

Finally, let us express our last main result by the following theorem. In the following theorem, $N(e)$ denotes the closed subgroup of the object group $G(e)$ of the unit element $e$. We define the set $M=\left\{a \circ N(e) \mid a \in S t_{G} e\right\}$. In fact, the set $M$ has a ring structure with the operations $(a \circ N(e))+(b \circ N(e))=(a+b) \circ N(e)$ and $(a \circ N(e))(b \circ N(e))=(a b) \circ N(e)$, where $a, b \in S t_{G} e$. Clearly, $M=S t_{R} e / N(e)$ and $M$ is a smooth manifold with quotient manifold structure. Since the ring operations of the Lie ring $R$ are smooth, the ring operations on $M$ are also smooth.

3.16. Theorem. Let $R$ be a transitive Lie ring-groupoid with the unit element e. Let $N(e)$ denote a closed Lie subgroup of $R(e)$. Under these conditions, we have naturally a Lie ring-groupoid $P$ and a covering morphism $p: P \rightarrow R$ of the Lie ring-groupoids such that the unit of $P_{0}$ is $\widetilde{e}=N(e)$. Furthermore, $p(P\{\widetilde{e}\})=N(e)$. 
Proof. By [6], there exists an action $\phi: R \alpha_{\alpha} \times{ }_{p} M \rightarrow M,(b, a \circ N(e)) \mapsto{ }^{b}(a \circ N(e))=$ $b \circ a \circ N(e)$ of the Lie groupoid $R$ on the manifold $M$ via $w: M \rightarrow R_{0}, a \circ N(e) \mapsto \beta(a)$. Also, since

$$
\begin{aligned}
w((a \circ N(e))(b \circ N(e))) & =w(a b \circ N(e))=\beta(a b)=\beta(a) \beta(b) \\
& =w(a \circ N(e)) w(b \circ N(e)),
\end{aligned}
$$

$w: M \rightarrow R_{0}$ is a Lie ring homomorphism. Therefore, the Lie ring-groupoid $R$ acts on the Lie ring $M$ via the smooth ring homomorphism $w$. Then, we obtain a Lie ringgroupoid $R \ltimes M$ with the object manifold $(R \ltimes M)_{0}=M$. Indeed; by [6], $R \ltimes M$ is a Lie group-groupoid. In addition, $R \ltimes M$ with the operation $\left(b, a^{\prime} \circ N(e)\right)(c, a \circ N(e))=$ $\left(b c,\left(a a^{\prime}\right) \circ N(e)\right)$ which is defined by the operation of the Lie ring $R$ is a Lie ring-groupoid.

Let us define $p: R \ltimes M \rightarrow R$ by $(b, a \circ N(e)) \mapsto b$ on the morphisms and by $w$ on the objects. Then, by [6], $p$ is a covering morphism of Lie group-groupoids. Also, since $p_{0}$ is given by $w$ and

$$
\begin{aligned}
p\left(\left(b, a^{\prime} \circ N(e)\right)(c, a \circ N(e))\right) & =p\left(\left(b c,\left(a^{\prime} a\right) \circ N(e)\right)=b c\right. \\
& =p\left(b, a^{\prime} \circ N(e)\right) p(c, a \circ N(e)),
\end{aligned}
$$

$p$ is a Lie ring homomorphism. Consequently, $p: R \ltimes M \rightarrow R$ is a covering morphism of Lie ring-groupoids. When $P=R \ltimes M$ and $\widetilde{x}=N(x)$ are taken, then it follows $p(P(\widetilde{x}))=N(x)$.

\section{References}

[1] Brown, R., Topology and Groupoids, BookSurge LLC, Deganwy, United Kingdom, 2006.

[2] Brown, R., Danesh-Naruie, G. and Hardy, J.P.L., Topological Groupoids II: Covering morphisms and G-spaces, Math. Nachr, 74, 143-145, 1976.

[3] Brown, R. and Spencer, C. B., G-groupoids, Crossed Modules and the Fundamental Groupoid of a Topological Group, Proc. Konn. Ned. Akad. v. Wet., 79, 196-302, 1976.

[4] Danesh-Naruie, G., Theory of Topological Groupoids, PhD. Thesis, The University of Southampton, England, 1970.

[5] Gabriel, P. and Zisman, M., Categories of Fractions and Homotopy Theory, Springer-Verlag, Heidelberg, 1967.

[6] Gursoy, M.H., Icen, I., Ozcan, A.F., Coverings and Actions of Structured Lie Groupoids, An. Ştiint. Univ. Al. I. Cuza Iaşi. Mat. (N.S.), 2, 661-679, 2016.

[7] Higgins,P.J., Categories and Groupoids, van Nostrand, New York, 1971.

[8] Icen, I., Gürsoy, M.H., Ozcan, A.F., Coverings of Lie Groupoids, Turk. J. Math., 35, 207$218,2011$.

[9] Icen, I., Ozcan, A.F. and Gursoy, M.H., Topological Group-Groupoids and Their Coverings, Indian J. pure appl. Math, 36 (9), 493-502, 2005.

[10] MacKenzie, K.C.H., General Theory of Lie Groupoids and Lie Algebroids, London Mathematical Society Lecture Note Series 213, Cambridge University Press, 2005.

[11] Moerdijk, I. and Mrcun, J., Introduction to Foliations and Lie Groupoids, Cambridge University Press, New York, 2003.

[12] Mucuk, O., Coverings and Ring-Groupoids, Georgian Math. J., Vol:5, No:5, 475-482, 1998.

[13] Ozcan, A.F., Icen, I. and Gursoy, M.H., Topological Ring-Groupoids and Liftings, Iranian Journal of Science and Technology, Transaction A, Vol. 30, No.A3, 2006. 